\title{
Constipation in ulcerative colitis: pathophysiology and practical management
}

\author{
Charles Miller (D) ,' Anton Emmanuel, ${ }^{1,2}$ Natalia Zarate-Lopez, ${ }^{1}$ \\ Stuart Taylor, ${ }^{3}$ Stuart Bloom ${ }^{2,4}$
}

\begin{abstract}
${ }^{1}$ Gastroenterology Department, University College London Hospitals NHS Foundation Trust, London, UK

${ }^{2}$ University College London, London, UK

${ }^{3} \mathrm{UCL}$ Centre for Medical Imaging, Charles Bell House, 43-45 Foley street, University College London, London, UK ${ }^{4}$ University College London Hospitals NHS Foundation Trust, London, UK
\end{abstract}

\section{Correspondence to}

Dr Charles Miller,

Gastroenterology, University

College London Hospitals NHS Foundation Trust, London NW1

2PG, UK; charles.miller@nhs.net

Received 30 May 2020

Revised 29 June 2020

Accepted 4 July 2020

Published Online First

26 August 2020

\section{Check for updates}

(C) Author(s) (or their employer(s)) 2021. No commercial re-use. See rights and permissions. Published by BMJ.

To cite: Miller $C$, Emmanuel $A$, Zarate-Lopez N, et al. Frontline Gastroenterology 2021;12:493-499.

\begin{abstract}
Clinical experience suggests that there is a cohort of patients with refractory colitis who do have faecal stasis that contributes to symptoms. The underlying physiology is poorly understood, partly because until recently the technology to examine segmental colonic motility has not existed. Patients are given little information on how proximal faecal stasis can complicate colitis. Treatment guidelines are scanty and many patients are offered little apart from laxatives and advice on increasing fibre intake, which often makes symptoms worse. This article aims to review the history, pathology and management, and create impetus for future research on this underappreciated condition.
\end{abstract}

\section{BACKGROUND}

The development of symptoms of constipation ('proximal constipation') is well recognised in patients with ulcerative colitis (UC), ${ }^{1-4}$ typically in the setting of active left-sided and distal disease. The presumed pathogenesis is faecal stasis proximal to the diseased segment, and uncomfortable symptoms of constipation can occur alongside symptoms of a UC flare. However, as constipation can occur in patients with pancolitis, as well as those with distal disease, ${ }^{5}$ the term proximal constipation may be misrepresentative. A previously proposed term 'ulcerative colitis-associated constipation' (UCAC) is more appropriate and will be adopted ${ }^{6}$ for the purposes of this review.

The concept of disease extent influencing the tendency to UCAC was recognised in the 1950s. Disease extent was noted to influence the stool form and consistency ${ }^{7}$ : when the disease was restricted to the distal colon, difficult voiding was seen more with normal rather than loose stool. A classic case series published in

\section{Significance of this study}

- Assessment of ulcerative colitis-associated constipation (UCAC) is currently inaccurate and abdominal radiograph (AXR) is not recommended; AXR transit studies may provide more useful information.

- Chronic inflammation has significant effects on the enteric nervous system, colonic structure and motility that may predispose to UCAC.

- Management currently is not evidence based and includes consideration of laxatives, prokinetics and biofeedback.

- Adequate treatment of UCAC may help achieve clinical remission in flares and may help avoid unnecessary treatment escalation.

- New diagnostic tools are required and cine MRI could play a future role.

the 1960s, when barium enema was a commonly used diagnostic tool, ${ }^{3}$ included six cases of left-sided colitis with evidence of faecal loading on barium study; in some cases, adequate treatment of constipation was required for successful treatment of the colitis. In one case, barium was retained for 5 weeks in the right side of the colon and in another the constipation caused subacute intestinal obstruction. Notably, the faecal loading was clinically unexpected in the majority of cases.

While the concept of UCAC is generally accepted, identification of UCAC based on symptoms alone may not be accurate. Tenesmus and left iliac fossa discomfort due to constipation can be confused with disease activity, and this may result in unnecessary investigation and treatment escalation. Constipation should always be considered in cases of refractory distal UC. ${ }^{18}$ Constipation symptoms occur in 
between a third and a half of patients with UC. ${ }^{69}$ There is no correlation with stool consistency or frequency in flaring patients with UC identified with constipation in transit studies. ${ }^{10}$ However, others have observed that constipation symptoms are present in a similar proportion of patients with quiescent disease. ${ }^{11}$ There is anecdotal, ${ }^{12}$ case series ${ }^{3}$ and study ${ }^{13}$ evidence that adequate treatment of UCAC can improve outcomes, including achieving clinical remission. In addition to symptomatic benefit and achieving remission, adequate treatment of UCAC may conceivably help avoid unnecessary treatment escalation to immunomodulators and biologics.

\section{Clinical presentation}

In the absence of an accepted definition for UCAC, diagnosis is usually made on clinical grounds coupled with a plain abdominal radiograph (AXR), but both these domains are of questionable value. A positive response to laxative treatment may also be suggestive.

\section{Symptoms and questionnaires}

UCAC is typically suspected in patients with a flare of left-sided UC with per rectal (PR) bleeding associated with symptoms suggestive of constipation with passage of hard stool, bloating, excessive flatus with the sensation of incomplete emptying. However, the overlapping symptoms of a flare and constipation including pain and tenesmus make diagnosis of UCAC on clinical grounds difficult. We know that stool consistency and frequency do not necessarily correlate with UCAC as demonstrated by transit study in UC flares, ${ }^{10}$ so judgement on symptoms alone is likely to be inaccurate. UCAC can occur in times of remission as well so the typical perception of the patient with UCAC may be incorrect.

Available definitions for constipation in the general population such as Rome III criteria have been adopted and modified to help define UCAC (box 1). ${ }^{6}$ The threshold symptom duration, with a minimum of 3 days a month, is low which could explain the high prevalence of $46 \%$ found compared with the previously mentioned studies. ${ }^{6}$ However, with these criteria, all patients had supportive AXR findings.

Box 1 Modified Rome III criteria for defining

ulcerative colitis-associated constipation: at least

two symptoms for $>3$ days for at least 3 months $^{6}$

Bloating

- Excessive or troublesome wind

- Abdominal cramping pain

- Reduced frequency of defecation compared with patient's own frequency

- Passage of hard or dry stool

- Straining at stool

- Sensation of incomplete defecation
Moreover, Rome IV criteria has superseded the previous version and other more useful tools are available for the assessment of constipation. ${ }^{14}$ The patient assessment of constipation symptoms (PAC-SYM) questionnaire is regularly used in research and may provide a better framework for a definition ${ }^{15}$. It has 12 symptom components each having a score weighting from 1 to 4 (absent to severe) that a patient has experienced within the last 2 weeks. This allows for a more detailed assessment of symptoms, particularly severity, as well as using patient-reported outcomes. Modification of this questionnaire with thresholds for symptom severity may be a more effective tool in identifying UCAC in clinical practice, research and assessing treatment response.

\section{Abdominal radiograph}

The AXR is the most routinely used investigation for UCAC but has significant limitations. It provides only a snapshot of faecal burden dependent on time of last meal and defecation, and provides no dynamic assessment of transit. Its performance in the assessment of constipation is unsatisfactory and its clinical utility questionable. ${ }^{16-18}$ One study demonstrated a marked disagreement between observers in up to $18 \%$ of AXRs viewed ${ }^{16}$; it used a formal scoring system ${ }^{19}$ for assessing faecal burden demarcating the colon into three segments (right colon, left colon and rectosigmoid colon) using anatomic landmarks, with a score of $0-5$ in each sector. Notably, even when used as a supportive tool, it does not correlate faecal loading with symptom severity. This is a time consuming and not routinely used assessment.

It is hard to justify repeated AXRs in clinical practice given the ionising radiation exposure and legislation attempting to protect patients from unnecessary exposure. ${ }^{20}$ Other diagnostic tools are very much needed.

\section{Pathophysiology of UCAC}

UC is increasingly considered a progressive disease with chronic inflammation causing accumulation of damage over time. ${ }^{21}$ Earlier aggressive treatment may avoid sequelae including dysmotility and propensity to the development of UCAC. The accumulation of gut wall structural damage and histological disturbance results in changes in the enteric nervous system and hence colonic motility. ${ }^{21-23}$

\section{Altered enteric nervous system}

The enteric nervous system is a complex regulatory system important in effective colonic motor function. It coordinates timed colonic propulsive smooth muscle contractions as well as segmental contractions, allowing passage of stool aborally.

In UC, the inflammation-driven morphological and quantitative changes may involve the interstitial cells of Cajal, ${ }^{21} 22$ the pacemaker cells that determine the maximal contraction frequency of each gut region. 
Myenteric ganglia are also morphologically altered in mouse models of UC. ${ }^{22}{ }^{24}$ Myenteric neurons have a critical role in peristalsis, ${ }^{25}$ so their injury is likely to alter motility and predispose to symptoms.

When exposed to inflammation enteric nervous system, abnormalities can persist even after recovery from inflammation, ${ }^{24}$ which may explain the tendency to UCAC even in times of remission.

\section{Altered colonic motility and transit in UC}

Motility and transit are clearly linked but are differing concepts, and in simple terms, represent physiological and clinical phenomena, respectively. Colonic motility is circadian in nature and more active in daylight hours. ${ }^{23}$ Colonic contents are moved predominantly by two types of contraction, the high amplitude propagated contractions (HAPC) and low amplitude propagated contractions (LAPC). Both are important in propulsion of colonic contents, with HAPCs being more important in propulsion of solid stool ${ }^{23}$ from the ascending colon to the rectosigmoid junction. HAPCs typically occur six times a day. ${ }^{26}$ There is also physiological retrograde propulsion of colonic contents, critical in water reabsorption, stool formation and continence maintenance.

In quiescent UC, manometric studies demonstrated an increase only in LAPCs compared with controls. ${ }^{27}$ By contrast in active UC, 24 hours manometry demonstrated an increased number of HAPC and LAPC compared with control, in a study where the majority had a left-sided disease distribution. ${ }^{28}$ A disordered gastrocolic reflex-a phenomenon mediated by a combination of autonomic and humoral factorshas been reported with reduced postprandial colonic motility in response to meals in patients with active left-sided UC. ${ }^{29}$

These findings suggest that motility differs in times of remission and active disease, which in turn could affect the tendency to UCAC. Considering that reduced HAPC is well recognised in slow transit constipation, ${ }^{30}$ the finding of increased HAPC in active UC is the likely substrate of the increased stool frequency and liquidity at such times. It is possible that in patients with UCAC during active phases, this increased contractility is ineffective in altering transit.

Colonic transit studies using markers and plain AXRs suggest that passage of markers is slow through the right side of the colon; however, passage through the diseased segment tends to be rapid. ${ }^{31}$ Remarkably, one study showed that a proportion of patients with active UC had total colonic transit times greater than 1 week using this methodology. ${ }^{12}$ Slow proximal transit has also been corroborated by radiotelemetry capsule studies in patients with UC with constipation where transit could take 5 days across the right side of the colon $^{32}$ and in patients with severe UC. ${ }^{33}$ Slowed proximal transit may not normalise once clinical remission has been achieved, again highlighting that disturbances can persist once inflammation has resolved. ${ }^{33}$

Demonstrable changes in colonic motility in patients with UC are not entirely consistent. ${ }^{21} 23$ This is likely related to case heterogeneity and small study numbers. A further factor for manometric studies is that catheters used in older studies were inaccurate as sensors were spaced too far apart, limiting spatial resolution of contractile activity. ${ }^{30}$

As with other painful anorectal conditions, puborectal dyssynergia may be a contributing factor to UCAC. Normal pelvic muscle function with coordinated contraction and relaxation is important in the process of effective defecation. This condition is well recognised in patients with inflammatory bowel disease (IBD) ${ }^{3435}$ and in one study $45 \%$ of patients were diagnosed with an evacuatory disorder. ${ }^{34}$

In summary, UCAC probably involves delayed rightsided transit in active UC resulting in a build-up of stool that becomes progressively more desiccated. The underlying motility issue is unclear, but may be related to reduced or ineffective/uncoordinated contractions, or increased number of retrograde contractions that result in impaired transit and development of UCAC.

\section{Consequences of disturbed motility and transit on drug} distribution

UCAC and altered motility may be part of the underlying mechanism in studies that suggest drug delivery to the diseased segment is impaired during disease activity. A study in active $\mathrm{UC}^{13}$ measured serum sulfapyridine levels as a proxy for adequate sulfalsalazine dosing. In patients who had satisfactory levels but ongoing endoscopically confirmed disease activity, all achieved clinical remission with the introduction of hydrophilic colloid and bran without a dosage change. The authors described these patients as 'constipated colitics'. The findings suggest that UCAC may impair delivery of the active metabolite to the inflamed distal colon with diminished therapeutic effect.

Regional distribution of orally administered eudragit-coated resin was examined in patients with active left-sided UC and healthy controls. ${ }^{36}$ It found higher levels in the right side of the colon in patients with UC (54\% vs 36\%) with significantly reduced levels in the left side of the colon (10\% vs 31\%) that suggests impairment in drug delivery in active disease.

\section{Investigation}

A palpable colon on clinical examination is not by itself evidence of constipation and the limited clinical utility of the AXR has already been discussed. Although rarely used for patients suspected to have constipation in UC, AXR colonic transit studies are readily available and provide an assessment on transit as well as a snapshot of faecal burden. Interpretation depends on methodology used, but movement of markers seems to be dependent on mass transit associated with HAPCs. 
However, transit studies have not been validated in IBD and the use of transit studies in this setting requires research. They are not used routinely at our institution currently.

Most other available tests have clear limitations and are not routinely used. CT can provide a snapshot of faecal burden but is not justifiable due to significant ionising radiation. Colonic scintigraphy can be used to assess transit; however, its availability is limited to specialist centres, requires multiple visits and exposure to ionising radiation. Colonic manometry remains a research tool and can give insights into colonic motility.

Telemetric capsule is not used routinely; it has been used to investigate intestinal transit in UC as well as acute severe UC (ASUC) ${ }^{32} 33$ but its use is limited by cost and availability.

Anorectal manometry (including high resolution) may provide important pathophysiological information on sphincter tone and rectoanal reflexes, thus identifying patients with dyssynergic defecation and guiding tailored biofeedback programmes. ${ }^{37}$

MRI is not currently routinely used but is being used increasingly in research to assess intestinal motility, ${ }^{38} 39$ including in constipation ${ }^{40}$ and assessment of treatment response in Crohn's disease. ${ }^{41} \mathrm{MRI}$ as an investigative technique is appealing in IBD given its lack of ionising radiation exposure and safety. The so-called 'Cine MRI' captures bowel motility at high temporal resolution (eg, 2 images/s) and provides dynamic assessment of regional and global motility. ${ }^{38} 42$ In addition to assessing faecal burden at a point in time, software is now available that can quantify regional luminal contents that could help objectively measure it in UCAC. ${ }^{42}$ There is the potential for an array of simultaneous assessments that could be performed including colonic motility, transit, colonic volume and contents. Currently, it has the potential to provide significant insights into the pathophysiology of UCAC in research and may have a role in future clinical practice. For example, with these data, we may be able to identify which patients may benefit from prokinetics rather than osmotic laxatives. If information from disease activity on the scan could be obtained simultaneously, then an invasive endoscopic evaluation may be avoided.

\section{Management}

Guidelines on managing UCAC are sparse. The British Society of Gastroenterology (BSG) makes mention of it being a common problem that it may contribute to treatment refractory proctitis. ${ }^{2}$ It suggests that functional bowel symptoms including constipation are common during remission. European Crohn's and Colitis Organisation (ECCO) similarly advise to assess for unrecognised constipation in refractory proctitis and distal colitis. ECCO recommends that an AXR maybe useful in assessment and if visible faecal loading then a laxative should be considered. ${ }^{1}$ There is not specific recommendation on diet, type or duration of laxative, or other drugs such as prokinetics in either guideline. There are no randomised controlled trials on the treatment of this condition.

Basic measures that may help include training to use the toilet at routine times, making use of the gastrocolic response when there is increased colonic motility, to attempt defecation approximately $30 \mathrm{~min}$ after meals or after waking. ${ }^{43}$

Increasing dietary fibre, including bulk forming laxatives can be considered. Though widely used, the role of increasing dietary fibre in the management of UCAC is not clear. Increased soluble fibre can be beneficial in other patient groups with constipation and may be beneficial in managing UCAC; however, the data for insoluble fibre are conflicting. ${ }^{3744}$ Sterculia has been used commonly in the management of UCAC, ${ }^{6}$ and there is some evidence that both bran and hydrophillic colloid in UCAC can help in achieving remission without the alteration of UC medication. ${ }^{13}$ Thus, a trial of ispaghula husk (or methylcellulose) in addition to dietary amendments may be a consideration.

Laxatives can be difficult to accept for a patient with a diarrhoea causing condition. This is especially the case if incorrectly diagnosed and a patient only experiences the side effects. There is no consensus on optimal duration and class of laxative. In an Australian study of 125 patients, the most commonly used laxative in patients with UCAC was macrogol and less frequently used were magnesium sulfate and picosulfate. ${ }^{6}$ In refractory distal colitis with suspected constipation, the use of one or two sachets of picolax or fleet phospho-soda has also been proposed. ${ }^{8}$ Ideally, therapeutic trials should be informed by physiology but this is not currently the case, and this a future clinical goal.

If the primary problem in UCAC is mechanical obstruction by colonic contents, then osmotic laxatives are likely to be most beneficial. Anecdotally, therapeutic trials of movicol can be effective. In this context, a high fibre diet may be unhelpful, and this may explain our centre's experience that increased fibre intake can have deleterious effects

If the primary problem is disordered motility, then prokinetics such as prucalopride, with a pan-enteric effect, may be a sensible treatment. In addition to prucalopride, bisacodyl also can induce HAPCs. ${ }^{45}{ }^{46}$ In the context of a current lack of a supportive diagnostic test, a trial short therapeutic trial could be considered as second line, or in conjunction with a typically used osmotic laxative. Intestinal secretagogues such as linaclotide may also be considered in laxative refractory cases, although there is no specific evidence for its use in UC. ${ }^{47}$

Defecatory disorders, including pelvic dyssynergia, are common in patients with $\mathrm{IBD}^{48}$ and must be considered especially when there is failure of mentioned management interventions. It is treatable with biofeedback that can alleviate pelvic floor muscle dysfunction and help with symptoms of constipation 
as well as other troublesome symptoms such as anal incontinence. $^{3435}$

There is a well-described but incompletely understood interplay between the gut and brain, and functional gut symptoms are common in IBD and should be considered as part of holistic management approach. This is especially true when there is a coexistent psychological disorder, but needs to be considered even when there is not. There is evidence for the effectiveness of low Fermentable Oligosaccharides, Disaccharides, Monosaccharides And Polyols (FODMAP) diet in the setting of IBD. ${ }^{49}$ Antidepressants and psychological therapies may offer benefit. ${ }^{50}$

Anecdotal recommendations for managing UCAC include the use of formulations of mesalazine such as olsalazine that may accelerate transit time. ${ }^{51}$

UCAC is recognised in the setting of ASUC. Plain abdominal X-ray is not a useful modality in this setting, either to assess disease extent or presence of proximal faecal loading. ${ }^{52}{ }^{53}$ However, there is a paucity of evidence in the effectiveness of its treatment. Furthermore, laxative therapy can complicate assessment of treatment response.

We suggest an outline of a management algorithm in figure 1.

\section{CONCLUSION AND FUTURE OUTLOOK}

UCAC is a well-recognised and common problem and currently hindered by issues with diagnosis and management. We lack a validated symptomatic definition, a sensitive and specific diagnostic tool, an understanding of the underlying pathophysiology and an evidence-based management strategy (see suggested research questions in box 2).

Although research and clinical investigation of UCAC is methodologically difficult, it is surprising this topic receives such limited attention in the literature,

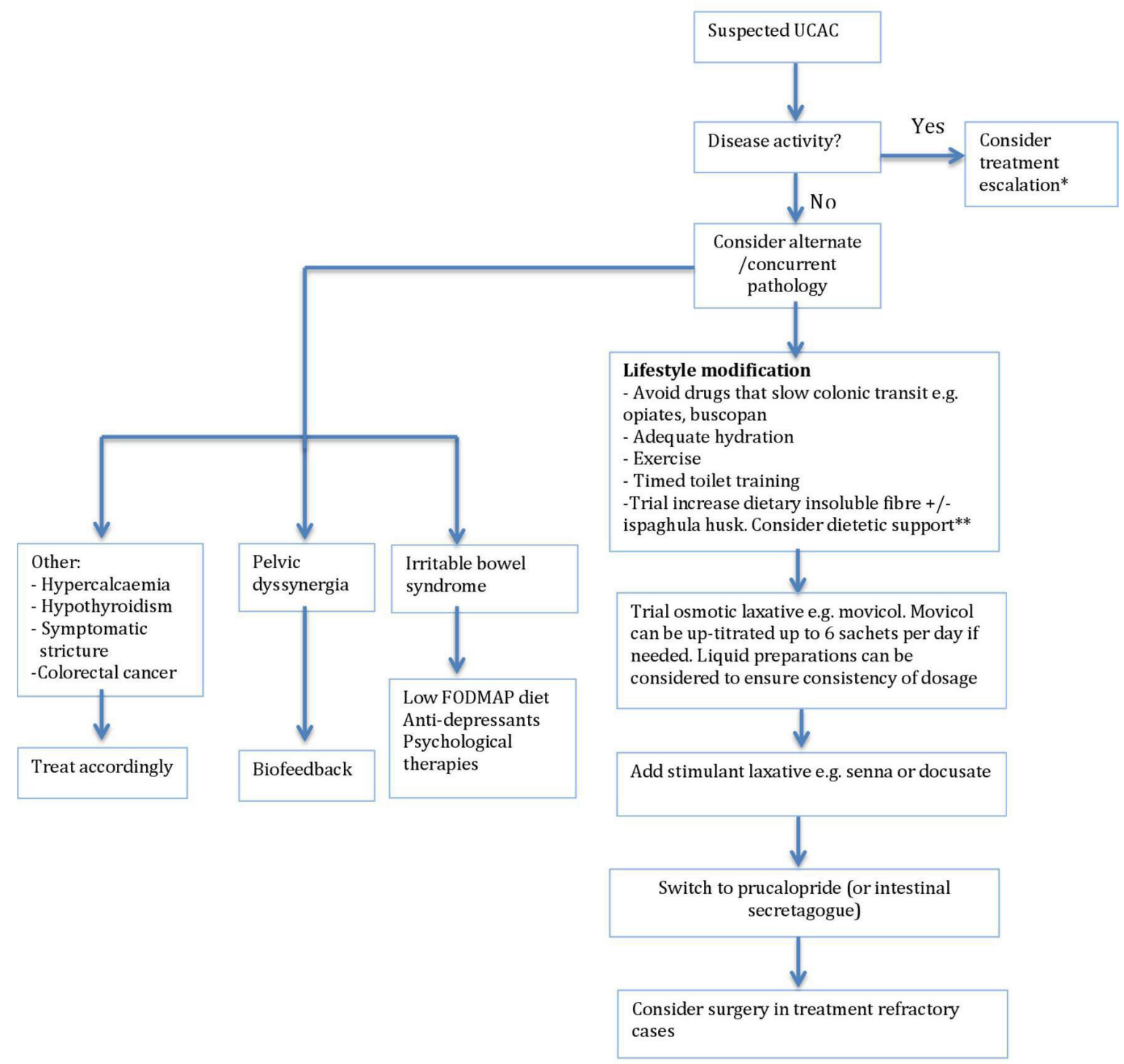

Figure 1 Outline of diagnostic differentials and management considerations in the management of ulcerative colitis-associated constipation (UCAC). * Treatment escalation may be avoidable with treatment of symptomatic UCAC. ${ }^{*}$ A trial of reduced fibre intake is also a consideration based on our centres experience. 


\section{Box 2 Future research questions include:}

1. What is the underlying motility disturbance in this condition?

2. What is the optimal diagnostic test to diagnose ulcerative colitis-associated constipation?

3. What is the optimal diet or dietary intervention for this condition?

4. What is the optimal laxative and pharmacological treatment strategy for this condition?

particularly as the issue has been previously shortlisted as an important research question in the IBD community. ${ }^{4}$

We would recommend studies into the use of questionnaires such as PAC-SYM in future attempt to devise asymptomatic definition, although a validated UCACdedicated questionnaire capturing the unique characteristic of the condition would be desirable. There is also a need to research correlations between symptom clusters and objective diagnostic findings to develop a validated clinical definition for UCAC.

The AXR is a non-specific and non-sensitive diagnostic marker for UCAC and we would not recommend its use. Given the current lack of viable alternative, colonic AXR transit studies could be a helpful tool for diagnosing UCAC but requires validation. It may give additional information on regional and total colonic transit, including pelvic dyssynergia. We propose that cine and quantitative MRI has great potential to improve understanding of the pathophysiology and for assessment and diagnosis of UCAC in future clinical practice. Concurrent assessment of disease activity may also be a possibility, avoiding endoscopic investigation.

Improved definitions and diagnostic methods of identifying constipation and assessing colonic motility would help characterise UCAC. With better identification and understanding of the pathology, we could better optimise treatment. It remains unclear which strategies of osmotic non-stimulant laxatives, stimulant laxatives, prokinetics or increasing dietary fibre provide optimal outcomes. The current practice of blind therapeutic trials is unsatisfactory.

Prevention and treatment of UCAC may improve aminosalicylate luminal distribution and efficacy in addition to symptomatic benefit. It is conceivable that there are patients who are escalated to thiopurines or even a biologic because this condition has not been suitably addressed. Preventing exposure to side effects of unnecessary medications as well as cost implications make satisfactory treatment appealing. How truly common and clinically important a problem this condition is yet to be ascertained. For now, it remains a headache in clinical practice.

Acknowledgements Belinda Theis for her thoughtful input, support and mastery of the English language.
Collaborators C P Miller; A Emmanuel; N Zarate-Lopez; S A Taylor; S L Bloom.

Contributors CM came up with the concept of the article and authored the article. SB came up with the concept of the article and gave critical review, made revisions and recommendations. SB is the senior author on the article. AE, NZ-L and ST made critical review of article, made revisions and recommendations.

Funding The authors have not declared a specific grant for this research from any funding agency in the public, commercial or not-for-profit sectors.

Competing interests ST is a shareholder in company Motilent. $\mathrm{He}$ is also a research consultant to Robarts.

Patient consent for publication Not required.

Provenance and peer review Not commissioned; externally peer reviewed.

\section{ORCID iD}

Charles Miller http://orcid.org/0000-0002-2068-4839

\section{REFERENCES}

1 Harbord M, Eliakim R, Bettenworth D, et al. Third European evidence-based consensus on diagnosis and management of ulcerative colitis. Part 2: current management. J CrohnsColitis 2017;11:769-84.

2 Lamb CA, Kennedy NA, Raine T, et al. British Society of gastroenterology consensus guidelines on the management of inflammatory bowel disease in adults. Gut 2019;68:s1-106.

3 Lennard-Jones JE, Langman MJ, Jones FA. Faecal stasis in proctocolitis. Gut 1962;3:301-5.

4 Hart AL, Lomer M, Verjee A, et al. What are the top 10 research questions in the treatment of inflammatory bowel disease? A priority setting partnership with the James Lind alliance. J Crohns Colitis 2017;11:204-11.

5 Jalan KN, Walker RJ, Prescott RJ, et al. Faecal stasis and diverticular disease in ulcerative colitis. Gut 1970;11:688-96.

6 James SL, van Langenberg DR, Taylor KM, et al. Characterization of ulcerative colitis-associated constipation syndrome (proximal constipation). JGH Open 2018;2:217-22.

7 Engel GL. Studies of ulcerative colitis I. clinical data bearing on the nature of the somatic process. Psychosom Med 1954;16:496-501.

8 Travis SPL. The management of refractory distal colitis. Journal of the Royal College of physicians of Edinburgh 2001;31:2.

9 Lennard-Jones JE, Cooper GW, et al. Observation in idiopathic ulcerative proctitis. Gut 1962;3:201-6.

10 Allison MC, Vallance R. Prevalence of proximal faecal stasis in active ulcerative colitis. Gut 1991;32:179-82.

11 Farrokhyar F, Marshall JK, Easterbrook B, et al. Functional gastrointestinal disorders and mood disorders in patients with inactive inflammatory bowel disease: prevalence and impact on health. Inflamm Bowel Dis 2006;12:38-46.

12 Stanfield C. Drug focus. consideration in the management of ulcerative colitis. Gastrointestinal Nursing 2020;14.

13 Cowan GO, Das KM, Eastwood MA. Further studies of sulphalsalazine metabolism in the treatment of ulcerative colitis.

14 McCrea GL, Miaskowski C, Stotts NA, et al. Review article: self-report measures to evaluate constipation. Aliment Pharmacol Ther 2008;27:638-48.

15 Frank L, Kleinman L, Farup C, et al. Psychometric validation of a constipation symptom assessment questionnaire. Scand J Gastroenterol 1999;34:870-7.

16 Cowlam S, Vinayagam R, Khan U, et al. Blinded comparison of faecal loading on plain radiography versus radio-opaque marker transit studies in the assessment of constipation. Clin Radiol 2008;63:1326-31.

17 Reber J, McGauvran A, Froemming A. Abdominal radiograph usage trends in the setting of constipation: a 10-year experience. Abdom Radiol 2018;43:2231-8. 
18 Driver BE, Chittineni C, Kartha G. Utility of plain abdominal radiogragh in adult ED patients with suspected constipation. Am J Emerg Med 2019;27:158377.

19 Leech SC, McHugh K, Sullivan PB. Evaluation of a method of assessing faecal loading on plain abdominal radiographs in children. Pediatr Radiol 1999;29:255-8.

20 The ionising radiation (medical exposure) regulations, 2017. Available: http://www.legislation.gov.uk/id

21 Torres J, Billioud V, Sachar DB, et al. Ulcerative colitis as a progressive disease: the forgotten evidence. Inflamm Bowel Dis 2012;18:1356-63.

22 Bernardini NSegnani C, Segnani C, Ippolito C, et al. Immunohistochemical analysis of myenteric ganglia and interstitial cells of Cajal in ulcerative colitis. J Cell Mol Med 2012;16:318-27.

23 Bassotti G, Antonelli E, Villanacci V, et al. Colonic motility in ulcerative colitis. United European Gastroenterol J 2014;2:457-62.

24 Mawe GM. Colitis-induced neuroplasticity disrupts motility in the inflamed and post-inflamed colon. J Clin Invest 2015;125:949-55.

25 Spencer NJ, Dinning PG, Brookes SJ, et al. Insights into the mechanisms underlying colonic motor patterns. J Physiol 2016;594:4099-116.

26 Bassotti G, De Roberto G, Castellani D. Normal aspects of colorectal motility and abnormalities in slow transit constipation. WJG 2005;11:2691-6.

27 Bassotti G, de Roberto G, Castellani D, et al. Normal aspects of colorectal motility and abnormalities in slow transit constipation. World J Gastroenterol 2005;11:2691-6.

28 Bassotti G, Villanacci V, Mazzocchi A, et al. Colonic propulsive and postprandial motor activity in patients with ulcerative colitis in remission. Eur J Gastroenterol Hepatol 2006;18:50710.

29 Reddy SN, Bazzocchi G, Chan S, et al. Colonic motility and transit in health and ulcerative colitis. Gastroenterology 1991;101:1289-97.

30 Dinning PG, Smith TK, Scott SM. Pathophysiology of colonic causes of chronic constipation. Neurogastroenterol Motil 2009;21:20-30.

31 Rao SS, Read NW, Brown C, et al. Studies on the mechanism of bowel disturbance in ulcerative colitis. Gastroenterology 1987;93:934-40.

32 Jalan KN, Walker RJ, Prescott RJ, et al. Faecal stasis and diverticular disease in ulcerative colitis. Gut 1970;11:68896.

33 Haase AM, Gregersen T, Christensen LA, et al. Regional gastrointestinal transit times in severe ulcerative colitis. Neurogastroenterol Motil 2016;28:217-24.

34 Tremaine WJ, Raffals LH, Bharucha AE, et al. 561 inflammatory bowel disease and Non-Relaxing pelvic floor dysfunction. Gastroenterology 2013;144:S1-4.

35 Perera LP, Ananthakrishnan AN, Guilday C, et al. Dyssynergic defecation: a treatable cause of persistent symptoms when inflammatory bowel disease is in remission. Dig Dis $\mathrm{Sci}$ 2013;58:3600-5.
36 Hebden JM, Blackshaw PE, Perkins AC, et al. Limited exposure of the healthy distal colon to orally-dosed formulation is further exaggerated inactive left-sided ulcerative colitis.

37 Camilleri M, Ford AC, Mawe GM, et al. Chronic constipation. Nat Rev Dis Primers 2017;3:17095.

38 de Jonge CS, Smout AJPM, Nederveen AJ, et al. Evaluation of gastrointestinal motility with MRI: advances, challenges and opportunities. Neurogastroenterol Motil 2018;30:e13257.

39 Hoad C, Clarke C, Marciani L, et al. Will MRI of gastrointestinal function parallel the clinical success of cine cardiac MRI? Br J Radiol 2019;92:20180433.

40 Lam C, Chaddock G, Marciani L, et al. Colonic response to laxative ingestion as assessed by MRI differs in constipated irritable bowel syndrome compared to functional constipation. Neurogastroenterol Motil 2016;28:861-70.

41 MRI for early response to anti-TNF therapy. IRSCTN14481560. Available: Https://doi.org/10.1186/ IRSCTN14481560

42 Major G, Murray K, Singh G, et al. Demonstration of differences in colonic volumes, transit, chyme consistency, and response to psyllium between healthy and constipated subjects using magnetic resonance imaging. Neurogastroenterol Motil 2018;30:e13400.

43 Rao SSC. Biofeedback therapy for constipation in adults. Best Pract Res Clin Gastroenterol 2011;25:159-66.

44 Suares NC, Ford AC. Systematic review: the effects of fibre in the management of chronic idiopathic constipation. Aliment Pharmacol Ther 2011;33:895-901.

45 Miner PB, Camilleri M, Burton D, et al. Prucalopride induces high-amplitude propagating contractions in the colon of patients with chronic constipation: a randomized study. Neurogastroenterol Motil 2016;28:1341-8.

46 Bharucha AE. High amplitude propagated contractions. Neurogastroenterol Motil 2012;24:977-82.

47 Lacy BE, Levenick JM, Crowell MD. Linaclotide: a novel therapy for chronic constipation and constipation-predominant irritable bowel syndrome. Gastroenterol Hepatol 2012;8:65360.

48 Rezaie A, Gu P, Kaplan GG, et al. Dyssynergic defecation in inflammatory bowel disease: a systematic review and metaanalysis. Inflamm Bowel Dis 2018;24:1065-73.

49 Gracie DJ, Hamlin PJ, Ford AC. The influence of the brain-gut axis in inflammatory bowel disease and possible implications for treatment. Lancet Gastroenterol Hepatol 2019;4:632-42.

50 Ford AC, Lacy BE, Harris LA, et al. Effect of antidepressants and psychological therapies in irritable bowel syndrome: an updated systematic review and meta-analysis. Am J Gastroenterol 2019;114:21-39.

51 Rao SS, Read NW, Holdsworth CD. Influence of olsalazine on gastrointestinal transit in ulcerative colitis. Gut 1987;28:14747.

52 Almer S, Bodemar G, Franzén L, et al. Plain x-ray films and air enema films reflect severe mucosal inflammation in acute ulcerative colitis. Digestion 1995;56:528-33.

53 Langmead L, Rampton DS. Plain abdominal radiographic features are not reliable markers of disease extent in active ulcerative colitis. Am J Gastroenterol 2002;97:354-9. 